\title{
Two-dipole model of the asymmetric Sun
}

\author{
Bertalan Zieger ${ }^{1,2, *}$ and Kalevi Mursula ${ }^{2}$ \\ ${ }^{1}$ Center for Space Physics, Boston University, 725 Commonwealth Avenue, Boston, MA 02215, USA \\ ${ }^{2}$ ReSoLVE Centre of Excellence, Space Climate Research Unit, University of Oulu, PO Box 3000, 90014 Oulu, Finland
}

Received 10 March 2020 / Accepted 28 July 2020

\begin{abstract}
The large-scale photospheric magnetic field is commonly thought to be mainly dipolar during sunspot minima, when magnetic fields of opposite polarity cover the solar poles. However, recent studies show that the octupole harmonics contribute comparably to the spatial power of the photospheric field at these times. Also, the even harmonics are non-zero, indicating that the Sun is hemispherically asymmetric with systematically stronger fields in the south during solar minima. We present here an analytical model of two eccentric axial dipoles of different strength, which is physically motivated by the dipole moments produced by decaying active regions. With only four parameters, this model closely reproduces the observed large-scale photospheric field and all significant coefficients of its spherical harmonics expansion, including the even harmonics responsible for the solar hemispheric asymmetry. This two-dipole model of the photospheric magnetic field also explains the southward shift of the heliospheric current sheet observed during recent solar minima.
\end{abstract}

Keywords: photospheric magnetic field / north-south asymmetry / spatial power spectrum / two-dipole model

\section{Introduction}

It is known since the early 20th century that sunspot activity is often different in the two solar hemispheres. A long period of stronger sunspot activity in the northern hemisphere occurred from the late 1950 s to early 1970 s, covering most of solar cycles 19 and 20. During the last several solar cycles, the northern hemisphere has been more active in the ascending phase of the solar cycle, and the southern hemisphere in the descending phase. This disparate evolution between the two solar hemispheres culminated during the ongoing cycle 24, where each of the two hemispheres formed its distinct maximum, separated in time by almost one and a half years.

Not only sunspots and other active regions (all different forms of the toroidal magnetic field) depict hemispheric asymmetry. The whole large-scale pattern of magnetic fields on the solar surface (photosphere) has also been found to be systematically north-south asymmetric (Zhao et al., 2005; Virtanen \& Mursula, 2014; Petrie, 2015; Zieger et al., 2019). This is particularly true for the solar polar fields, which are unipolar around solar minima. Polar fields develop when the magnetic fields of decaying active regions that were generated at lower latitudes, are transported to high latitudes. Therefore, the asymmetry of polar fields naturally reflects asymmetry in poloidal magnetic field generation and/or transport. Using polar faculae as proxies of the polar fields, Goel \& Choudhuri (2009) estimated the

\footnotetext{
*Corresponding author: berci@bu. edu
}

hemispheric asymmetry at solar minima during the 20th century. While the asymmetry of polar fields was negative (southern hemisphere dominance) at the beginning of solar cycles 21-24, it was predominantly positive (northern hemisphere dominance) at the beginning of the earlier solar cycles 15-20, which is more or less in agreement with the observed hemispheric asymmetry in the total sunspot area during these solar cycles.

Measuring the polar fields in the photosphere is challenging because of the small line-of-sight component of the magnetic field at high solar latitudes. In addition, the solar poles are not equally visible during most of the year because of the tilt of the solar rotation axis with respect to the ecliptic, which is commonly referred to as the vantage point effect. The authors have recently developed a new method of eliminating the vantage point effect in photospheric magnetic field observations, which allows a more accurate calculation of the spatial power spectrum during solar minima (Zieger et al., 2019). It was shown that the zonal octupole component has a large contribution to the spatial power of the photospheric magnetic field during solar minimum conditions, matching the power of the dipole component. Also, the power of the low-degree even zonal harmonics was found to be statistically significant (Zieger et al., 2019), confirming the north-south asymmetry of the large-scale photospheric magnetic field.

Several studies have verified by now that the southern polar fields were stronger than the northern fields around solar minima during the recent solar cycles (21-24). This difference 

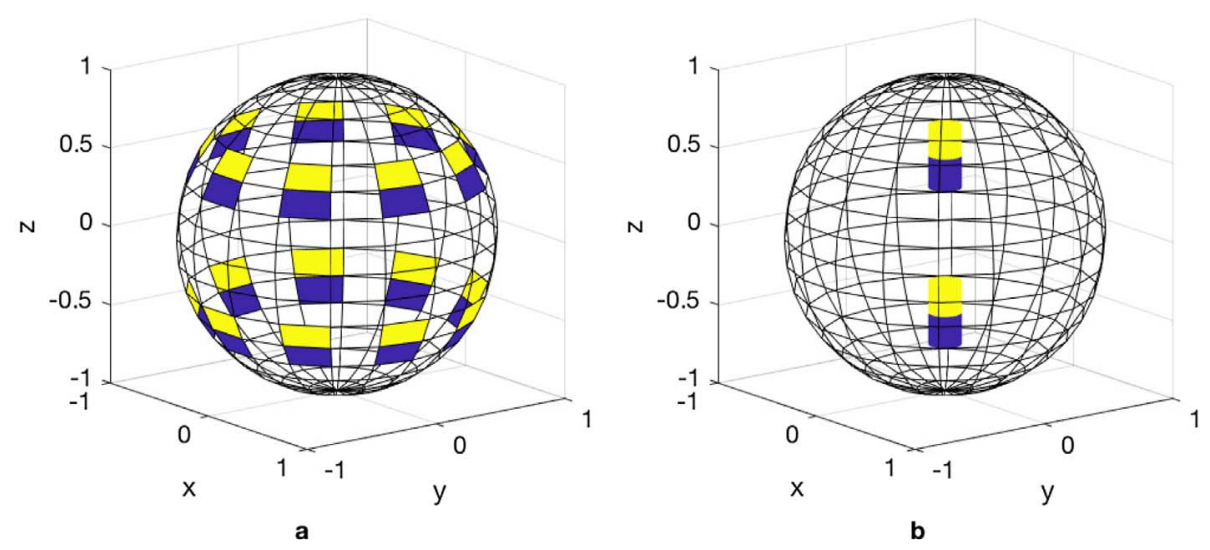

Fig. 1. Two-dipole model. (a) Two rings of surface dipoles representing the north-south magnetic component of decaying active regions in the photosphere at the latitude of about $\pm 30^{\circ}$ in an axisymmetric case. (b) Two axial dipoles placed at the center of each dipole ring of panel a. The magnetic potential of the two dipole rings of panel a and of the two axial dipoles of panel $b$ are nearly identical at the solar surface.

gives one of the most tangible evidence for solar hemispheric asymmetry that is systematically repeated from one solar minimum to the next. The detailed harmonic structure of the photospheric magnetic field, including its systematic hemispheric asymmetry, cannot yet be explained by current solar dynamo models (for a review, see Charbonneau, 2010). However, neither the global structure of the photospheric field nor its hemispheric asymmetry is only of academic or theoretical interest. They affect the distribution of solar eruptions, flares and coronal mass ejections, as well as the occurrence of high-speed solar wind streams. They have, therefore a great practical significance for space weather observed at the Earth.

In this paper we present a novel, analytical model of the large-scale photospheric magnetic field, which very accurately reproduces the observed distribution of photospheric magnetic fields during solar minima, and naturally explains the observed hemispheric asymmetry. We introduce the model in Section 2 and validate it with photospheric magnetic field data in Section 3. The modeling results of the photospheric and coronal north-south asymmetry are discussed in Section 4 . We present our conclusions in Section 5.

\section{Two-dipole model}

Sunspots and other active regions are concentrations of strong magnetic fields, which appear at low to mid-latitudes on the solar surface in pairs of opposite magnetic polarity. Sunspot pairs follow Hale's law, according to which the polarity of the leading spots in one hemisphere is opposite that of the leading spots in the other hemisphere and the polarities reverse from one solar cycle to the next. Thus, two solar cycles make one 22-year Hale cycle of the solar magnetic field (Hale, 1924). The leading spots tend to appear slightly closer to the equator than the trailing spots, a phenomenon known as Joy's law (Hale et al., 1919; Howard, 1991; Wang \& Sheeley, 1991; Sivaraman et al., 1999; Dasi-Espuig et al., 2010). During the rising phase of the solar cycle, the trailing spots have an opposite magnetic polarity compared to the polar fields in the same hemisphere during the previous minimum. Thus, as the trailing fields of decaying active regions are transported towards the pole (Wang et al., 1989; Hathaway \& Rightmire, 2010), they reverse the polar field around sunspot maximum. Accordingly, solar polar fields change polarity from one minimum to another, following the 22-year magnetic cycle. Joy's law and the poleward transport of trailing fields produce a north-south (poloidal) component of the magnetic field in the declining to minimum phase of the sunspot cycle. This was the observational motivation for the flux transport dynamo model introduced by Babcock (1961) and Leighton (1964, 1969). These empirical laws regulating the spatial distribution and temporal evolution of the photospheric magnetic field form the physical basis of the two-dipole model.

The trailing fields that are transported poleward and the leading fields that are transported equatorward can be represented as a set of magnetic dipoles (ideally a ring of dipoles) in each hemisphere, located at a typical latitude of active region generation, as shown in Figure 1a for an ideal, axisymmetric case. The authors would like to point out that this ring of dipoles is not equivalent mathematically with the double ring of opposite radial fluxes introduced as source terms in some early axisymmetric dynamo simulations (Durney, 1995; Nandy \& Choudhuri, 2001) to mimic the Babcock-Leighton poloidal magnetic flux emergence. The authors believe that it is more physical to represent decaying bipolar active regions with their dipole moments, which does not violate the divergence-free nature of the magnetic field and does not introduce artificial discontinuities in the magnetic potential.

The combined magnetic potential of several dipoles along the solar circumference can be closely approximated by a virtual north-south oriented (axial) dipole that is placed in the middle of the dipole ring as shown in Figure 1b. Although the individual dipoles in Figure 1a possess non-zonal harmonic terms as well, these terms vanish at the limit of axisymmetry when the number of dipoles approaches infinity. Because the polarities of the leading and trailing fields are oppositely ordered in the two hemispheres by Hale's law (Hale, 1924), the dipole moments of the two axial dipoles in the northern and southern hemisphere in Figure 1b should point in the same direction. The superposition of the two dipole fields reduces the photospheric magnetic field at low latitudes and enhances the magnetic field at high latitudes, which leads to strong polar 

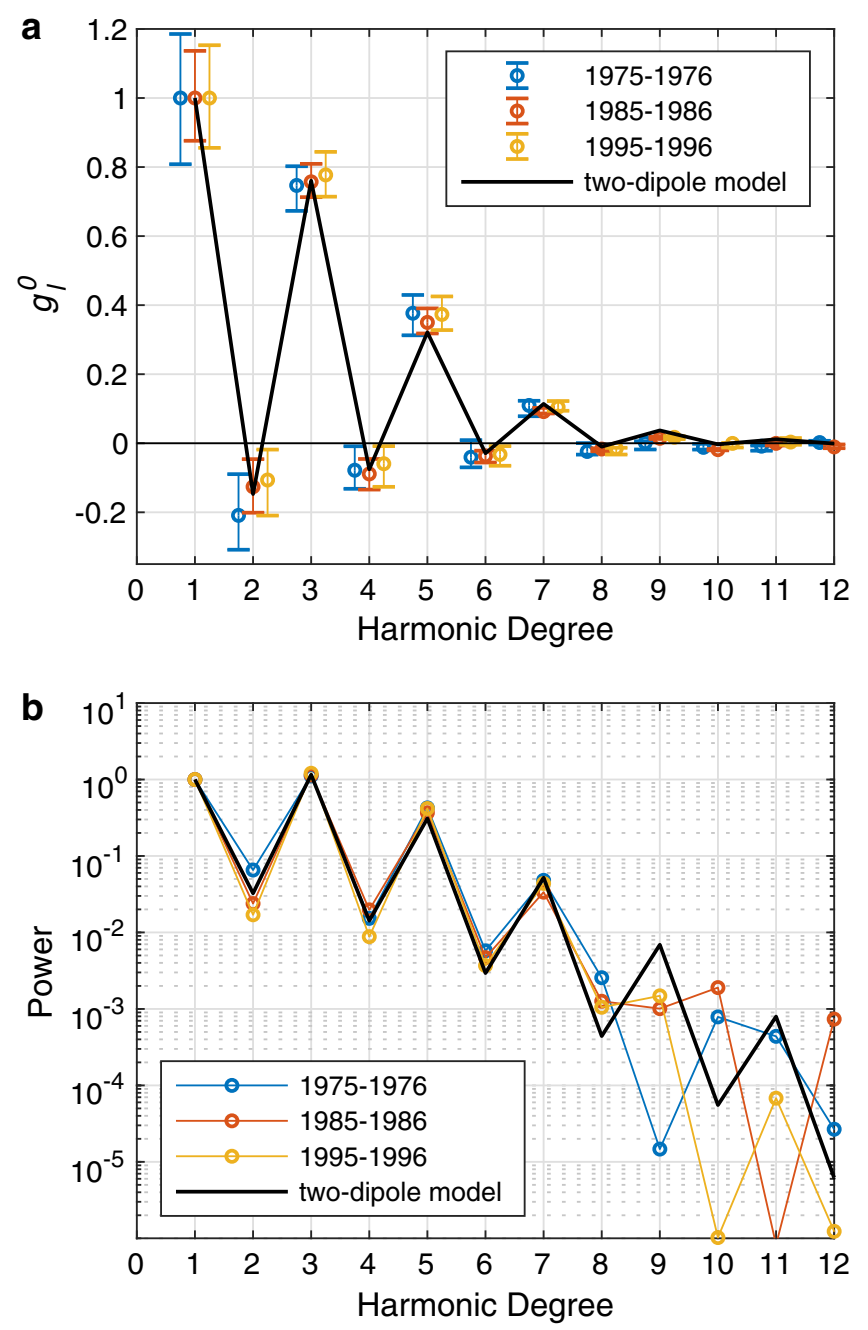

Fig. 2. Spherical harmonic structure of the photospheric magnetic field. (a) Normalized zonal Gauss coefficients of the photospheric magnetic potential for three consecutive solar minima. The twodipole model obtained from the first four Gauss coefficients is shown in black. (b) Normalized zonal spatial power spectra of the photospheric magnetic field for the same solar minima as in panel a, showing the relative contributions of the different harmonics (multipoles) to the total power.

fields, as demonstrated in Figure 3. We note that there is an analytical solution for the magnetic potential produced by such an eccentric dipole (Hurwitz, 1960; Winch, 1968), which greatly facilitates the calculation of the combined magnetic potential produced by the many surface sources.

In an axially symmetric case of Figure 1b, only the zonal Gauss coefficients $g_{l}^{0}$ of the spherical harmonics expansion of the magnetic potential are non-zero. We find the following form for $g_{l}^{0}$ for the eccentric two-dipole model (see detailed derivation in Appendix):

$$
g_{l}^{0}=l a_{1} z_{1}^{l-1}+l a_{2} z_{2}^{l-1},
$$

where $l$ is the spherical harmonic degree, $a_{1}$ and $a_{2}$ are the strengths of the two dipoles, and $z_{1}, z_{2}$ are their locations along the $z$-axis of symmetry (effectively the latitudes of the

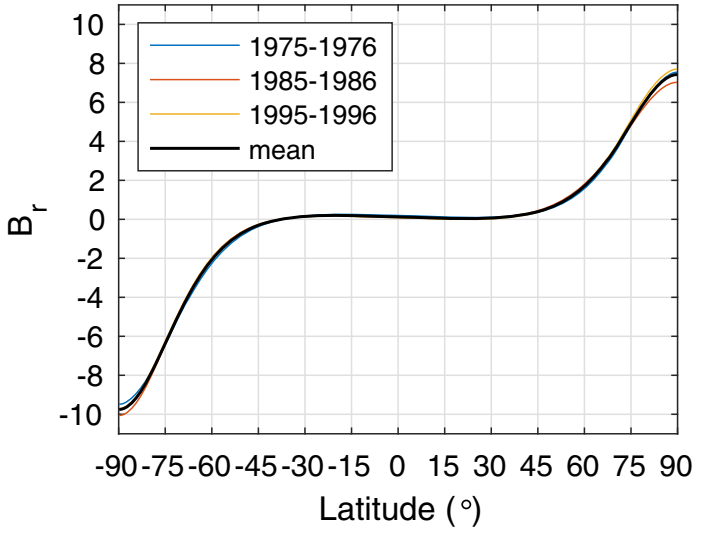

Fig. 3. Latitudinal distribution of the photospheric radial magnetic field obtained from the best-fitting two-dipole model for each of the three solar minima (1975-1976, 1985-1986 and 1995-1996). The radial magnetic field has been normalized to the signed dipole term $\left(g_{1}^{0}\right)$ at each solar minimum to allow direct comparison. The mean latitudinal profile is plotted in black.

dipole rings). Note that for a north-south symmetric model ( $a_{1}=a_{2}, z_{1}=-z_{2}$ ), equation (1) gives zero for all even-degree coefficients. Non-zero even-degree harmonics can be obtained only by breaking the hemispheric symmetry either having different strengths $\left(a_{1} \neq a_{2}\right)$ or distances $\left(\left|z_{1}\right| \neq\left|z_{2}\right|\right)$ for the two dipoles.

\section{Model validation with photospheric magnetic field data}

In order to estimate the success of the model of two eccentric dipoles, we compare the model prediction to the spherical harmonics expansion of the photospheric magnetic potential. We use here the synoptic maps of the photospheric radial magnetic field measured at the Mount Wilson Observatory (MWO) during two-year periods of three solar minima in 1975-1976, 1985-1986, and 1995-1996. The large-scale photospheric magnetic field during solar minima is axisymmetric and dominated by a few low harmonics (DeRosa et al., 2012; Petrie, 2013; Zieger et al., 2019). Therefore, it is sufficient to use only the lowest zonal Gauss coefficients (here up to $l=24$ ) of the magnetic potential. The method of deriving the zonal Gauss coefficients from the synoptic maps of the radial magnetic field and estimating their error is described in detail in Zieger et al. (2019). Figure 2 presents the zonal Gauss coefficients and the zonal spatial power spectra (for definition, see Zieger et al., 2019) for the MWO photospheric magnetic field as well as for the two-dipole model field during these three solar minima. The Gauss coefficients and the power spectra are normalized by the dipole term $(l=1)$ of the respective solar minimum so that the relative strengths of the harmonics can be compared among the three minima with different overall magnetic powers (Smith \& Balogh, 2008; Zhou \& Smith, 2009). Note that the (normalized) Gauss coefficients for the three minima agree with each other (at least up to $l=8$ ) within the estimated errors. This shows that the distribution of magnetic fields on the solar surface remains closely 

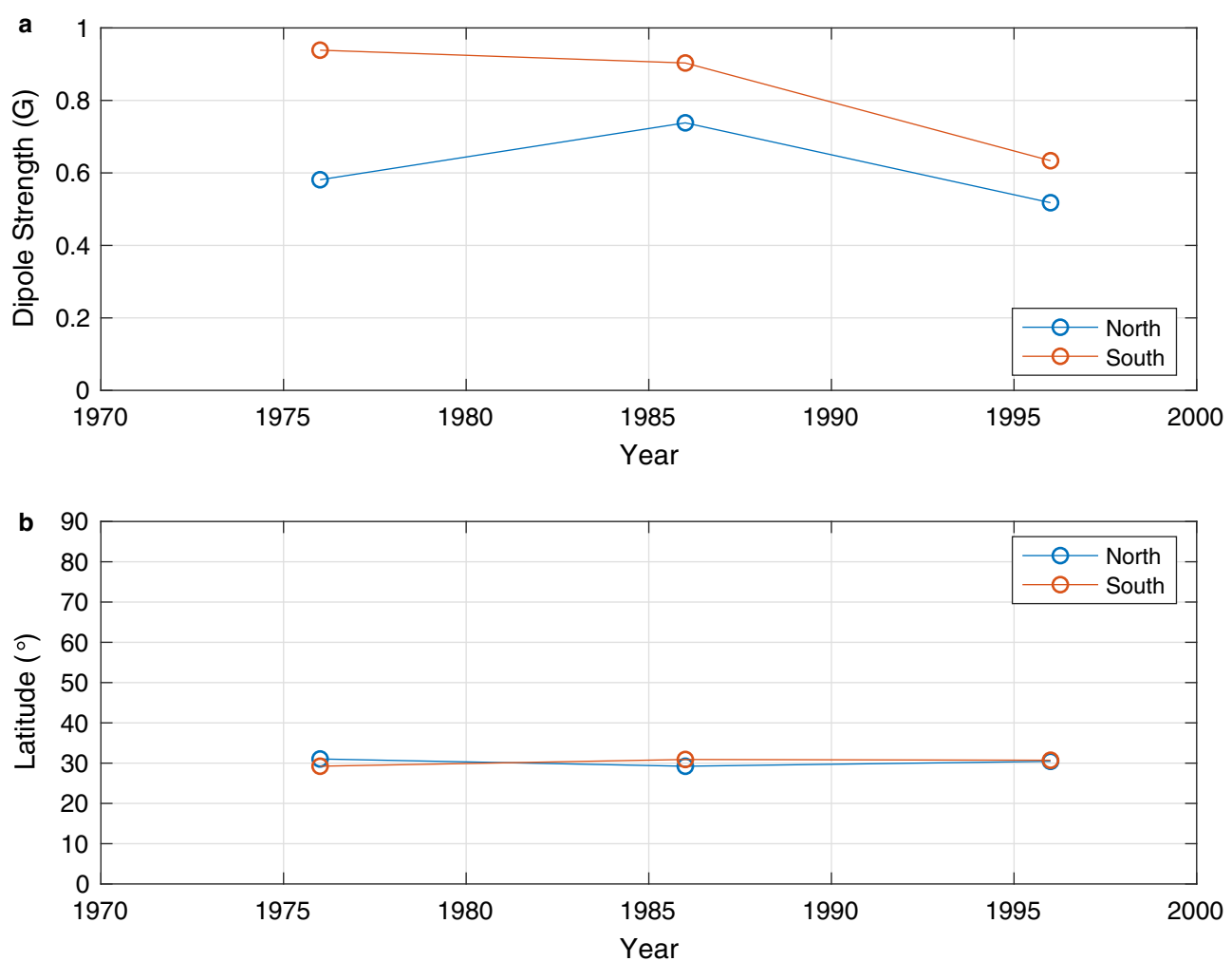

Fig. 4. Parameters of the northern and southern dipoles. (a) Strengths of the northern and southern dipole for the three solar minima (19751976, 1985-1986 and 1995-1996). The southern dipole is stronger than the northern dipole during all three solar minima. (b) Locations of the northern and southern dipoles in solar latitude (southern latitudes are reversed to positive).

similar from one solar minimum to the next, despite different total powers.

The two-dipole model has four parameters $\left(a_{1}, a_{2}, z_{1}, z_{2}\right)$, which are exact solutions of equation (1). They depend on the first four zonal Gauss coefficients as follows:

$$
\begin{aligned}
z_{1}= & \left(9 g_{1}^{0} g_{4}^{0}-6 g_{2}^{0} g_{3}^{0}+\sqrt{3}\left(27\left(g_{1}^{0}\right)^{2}\left(g_{4}^{0}\right)^{2}\right.\right. \\
& -108 g_{1}^{0} g_{2}^{0} g_{3}^{0} g_{4}^{0}+64 g_{1}^{0}\left(g_{3}^{0}\right)^{3}+54\left(g_{2}^{0}\right)^{3} g_{4}^{0} \\
& \left.\left.-36\left(g_{2}^{0}\right)^{2}\left(g_{3}^{0}\right)^{2}\right)^{1 / 2}\right) /\left(24 g_{1}^{0} g_{3}^{0}-18\left(g_{2}^{0}\right)^{2}\right), \\
z_{2}= & \left(2 g_{3}^{0}-3 g_{2}^{0} z_{1}\right) /\left(3 g_{2}^{0}-6 g_{1}^{0} z_{1}\right), \\
a_{1}= & \left(g_{2}^{0}-2 g_{1}^{0} z_{2}\right) /\left(2 z_{1}-2 z_{2}\right), \\
a_{2}= & g_{1}^{0}-a_{1} .
\end{aligned}
$$

We have used the average values of the first four Gauss coefficients of the synoptic maps during the three minima to derive the values of these four parameters. Once the four model parameters were fixed, we calculated the higher-degree coefficients (up to $l=24$ ) of the two-dipole model using equation (1). This two-dipole model was then plotted in black in Figures $2 \mathrm{a}$ and $2 b$. Figure 2 shows that the two-dipole model yields Gauss coefficients and power spectra that have an excellent agreement with observations. Moreover, Figure $2 \mathrm{~b}$ shows that the octupole component $(l=3)$ has even a slightly higher power in the power spectrum than the dipole component $(l=1)$.

As seen in Figure $2 b$, the observed spatial power spectra during the three solar minima are similar up to $l=8$ but become uncorrelated for higher degrees $(l>8)$. This suggests that the MWO observations are dominated by noise beyond $l=8$, in agreement with earlier results (Zieger et al., 2019). Figure $2 b$ also shows that the two-dipole model closely reproduces the observed power spectrum until $l=8$. Accordingly, the twodipole model yields a close representation of the axisymmetric magnetic field during solar minima, reproducing all the statistically significant zonal Gauss coefficients $(l<9)$ that are responsible for most of the spectral power.

\section{Modeling the north-south asymmetry in the photosphere and in the corona}

Most of the low even-degree Gauss coefficients $(l=2,4,6,8)$ in Figure 2a are small but significantly different from zero during the three solar minima. This means that the photospheric magnetic field is north-south asymmetric during these solar minima. The fact that all significant even-degree coefficients are negative implies that these terms weaken the northern polar field but strengthen the southern polar field. Thus, Figure $2 \mathrm{a}$ verifies the earlier result that the southern solar polar magnetic fields were stronger than the northern fields during the recent 
solar minima (Zhao et al., 2005; Erdós \& Balogh, 2010; Virtanen \& Mursula, 2010; Petrie, 2015).

In order to study this hemispheric asymmetry in more detail, we fitted the four parameters of the two-dipole model to the first four Gauss coefficients individually for each solar minimum. The latitudinal profiles of the photospheric radial magnetic field obtained from the first 24 Gauss coefficients of the two-dipole model is plotted in Figure 3 for the three solar minima. We normalized the radial magnetic field to the dipole term $\left(g_{1}^{0}\right)$ so that the three solar minima would be directly comparable. The mean of the three radial magnetic field profiles is shown in black. The latitudinal distribution of the radial magnetic field is very similar for the three solar minima, showing a clear asymmetry with a southern polar field $\sim 31 \%$ stronger than the northern polar field.

The strengths of the northern and southern axial dipoles (parameters $a_{1}$ and $a_{2}$ ) for the three minima are shown in Figure $4 \mathrm{a}$ and the latitudinal locations of the dipoles (parameters $z_{1}$ and $\left|z_{2}\right|$ converted to solar latitude) in Figure 4b. Figure 4b shows that the latitudes of the northern and southern dipoles are almost exactly the same, about $30^{\circ}$, which is a typical latitude of emerging active regions in the beginning of the solar cycle (Petrie, 2013). Accordingly, flux emergence is roughly symmetric with respect to the equator. Moreover, the fact that the dipole latitudes remain the same from one solar minimum to another suggests that the spatial location of processes producing new flux to the surface remains constant during time scales of, at least, a few solar cycles.

Figure 4a shows that the strength of the southern axial dipole is systematically stronger than the northern dipole during the three solar minima. Accordingly, the southern polar field is stronger than the northern polar field during solar minima, which confirms earlier results (Zhao et al., 2005; Erdős \& Balogh, 2010; Virtanen \& Mursula, 2010; Petrie, 2015). The southern dipole was $62 \%$ stronger than the northern dipole in 1975-1976, but only $22 \%$ stronger in 1985-1986 and 19951996. Based on the results depicted in Figure 4, it is clear that the observed hemispheric asymmetry of the photospheric magnetic field is mainly due to the different strengths, not due to different locations of decaying active regions in the two hemispheres. We also note that a single eccentric axial dipole can produce a north-south asymmetric magnetic field and explain the dipole $(l=1)$ and quadrupole $(l=2)$ Gauss coefficients (Wang, 1996; Pulkkinen et al., 1999; Mursula \& Hiltula, 2004; Zhao et al., 2005; Virtanen \& Mursula, 2016), but cannot reproduce the higher-degree Gauss coefficients, especially the observed strong octupole moment $(l=3)$.

An asymmetric photospheric field also affects the largescale structure of the solar coronal magnetic field. We have investigated this by computing the potential-field source-surface (PFSS) model of the coronal field (Wang \& Sheeley, 1992) between the solar surface and the coronal source surface at 2.5 solar radii, using the magnetic field of the two-dipole model as an inner boundary condition. The coronal magnetic field corresponding to the average two-dipole model for the three minima is depicted in Figure 5, where we have also included the MWO photospheric field observed during Carrington rotation 1910 in year 1996, as a typical example of photospheric magnetic field distribution during solar minimum. The dashed circle at 2.5 solar radii marks the source surface, where the coronal magnetic field becomes radial in the PFSS model.

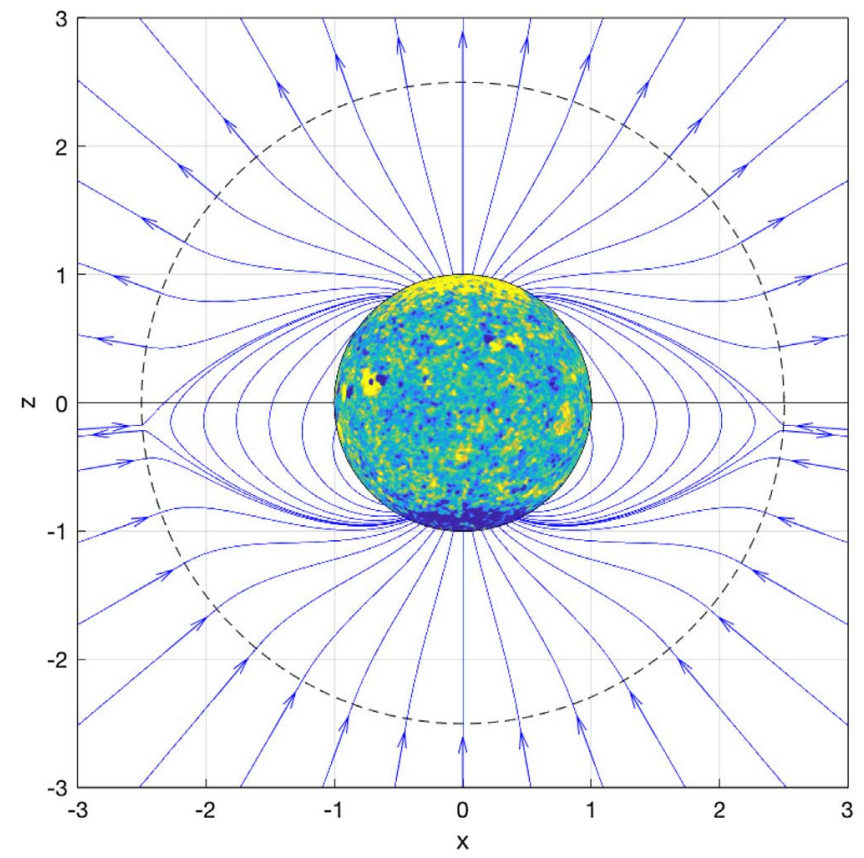

Fig. 5. Coronal magnetic field from the two-dipole model. Average coronal magnetic field during the three solar minima obtained from the two-dipole model of the photospheric field using the PFSS model. The coronal source surface, where magnetic field lines (in blue) become purely radial is marked with a dashed circle. The photospheric radial magnetic field observed during Carrington rotation 1910 in year 1996 is shown on the solar surface, featuring typical unipolar regions at the poles, with yellow and blue colors indicating positive (away from the Sun) and negative (toward the Sun) magnetic polarity, respectively. The heliospheric current sheet is shifted towards the south by $4.1^{\circ}$.

Figure 5 shows that there is a significant north-south asymmetry also in the large-scale structure of the solar corona. Most notably, the heliospheric current sheet (HCS), the magnetic equator outside the source surface (also called the ballerina skirt), where the field lines of opposite polarity meet each other (see Fig. 5), is shifted or coned southwards. Note also that the magnetic field lines forming closed loops in the inner corona are also asymmetric with respect to the solar equator. Interestingly, some of the closed field lines at low southern latitudes are anchored to the southern hemisphere at both ends. The southward shift of the HCS is in a good agreement with earlier results using the heliospheric magnetic field observed near the Earth (Mursula \& Hiltula, 2003; Koskela et al., 2018) and by the Ulysses probe (Erdős \& Balogh, 2010; Virtanen \& Mursula, 2010). We find that the average shift angle of the HCS for the three minima is $4.1^{\circ}$, and varies from $5.5^{\circ}$ for the first minimum to $3.6^{\circ}$ for the second and $3.0^{\circ}$ for the last minimum. These estimates for the southward shift of the HCS agree with an earlier estimate of HCS shift in the corona (Koskela et al., 2018) and support the result that the HCS shift in the corona is larger than the shift observed further out in the heliosphere (Mursula \& Hiltula, 2003; Erdős \& Balogh, 2010; Virtanen \& Mursula, 2010; Koskela et al., 2018). The decreasing HCS shift suggests that the asymmetry was larger during the earlier, stronger cycles, and has decreased with the reduction of the overall 
solar activity. Accordingly, the north-south asymmetry of the photospheric magnetic field is expected to be the smallest in the beginning of low solar activity cycles like solar cycle 24 . As mentioned in Section 1, solar cycle 24 was exceptional in many ways, therefore we did not include it in this study, but it will be subject of future work.

\section{Conclusions}

We have shown here that the large-scale structure of the photospheric magnetic field during solar minima can be closely described with an analytical model consisting of two eccentric axial dipoles that represent the combined magnetic potential of decaying active regions. We find that, while the effective location of the dipoles is the same in the two hemispheres, the southern dipole is systematically stronger than the northern dipole during recent solar minima. This difference explains the observed north-south asymmetry in the solar polar fields and in the heliospheric current sheet location. Our analytical twodipole model also offers a new method for reconstructing the poorly observed or missing values of the solar polar fields using reliably measured lower-latitude observations, which is critical for more accurate predictions of future solar activity (Choudhuri et al., 2007; Hathaway \& Upton, 2016), space weather, and space climate. This novel polar field filling method is based on an iterative procedure where the Gauss coefficients and the high-latitude solar magnetic fields are updated in each iteration step until the polar field converges to a constant value. More specific details on this iterative procedure will be given in a follow-up paper.

Acknowledgements. The authors acknowledge the financial support by the Academy of Finland to the ReSoLVE Centre of Excellence (Project No. 307411). Mt. Wilson data are publicly available at ftp://howard.astro.ucla.edu/pub/obs/ synoptic_charts/fits/ courtesy of Roger Ulrich. The editor thanks two anonymous reviewers for their assistance in evaluating this paper.

\section{References}

Babcock HW. 1961. The topology of the Sun's magnetic field and the 22-year cycle. Astrophys J 133: 572. https://doi.org/10.1086/ 147060 .

Charbonneau P. 2010. Dynamo models of the solar cycle. Living Rev Sol Phys 7(1): 3. https://doi.org/10.12942/lrsp-2010-3.

Choudhuri AR, Chatterjee P, Jiang J. 2007. Predicting solar cycle 24 with a solar dynamo model. Phys Rev Lett 98(13): 131103. https://doi.org/10.1103/PhysRevLett.98.131103.

Dasi-Espuig M, Solanki SK, Krivova NA, Cameron R, Peñuela T. 2010. Sunspot group tilt angles and the strength of the solar cycle. A\&A 518: A7. https://doi.org/10.1051/0004-6361/201014301.

DeRosa ML, Brun AS, Hoeksema JT. 2012. Solar magnetic field reversals and the role of dynamo families. Astrophys $J$ 757(1): 96. https://doi.org/10.1088/0004-637X/757/1/96.

Durney BR. 1995. On a Babcock-Leighton dynamo model with a deep-seated generating layer for the toroidal magnetic field. Sol Phys 160(2): 213-235. https://doi.org/10.1007/BF00732805.
Erdős G, Balogh A. 2010. North-south asymmetry of the location of the heliospheric current sheet revisited. J Geophys Res 115(A1): A01105. https://doi.org/10.1029/2009JA014620.

Goel A, Choudhuri AR. 2009. The hemispheric asymmetry of solar activity during the last century and the solar dynamo. Res A\&A 9 (1): 115-126. https://doi.org/10.1088/1674-4527/9/1/010.

Hale GE. 1924. Sun-spots as magnets and the periodic reversal of their polarity. Nature 113(2829): 105-112. https://doi.org/10. 1038/113105a0.

Hale GE, Ellerman F, Nicholson SB, Joy AH. 1919. The magnetic polarity of sun-spots. Astrophys J 49: 153. https://doi.org/10.1086/ 142452.

Hathaway DH, Rightmire L. 2010. Variations in the Sun's meridional flow over a solar cycle. Science 327(5971): 1350. https://doi. org/10.1126/science.1181990.

Hathaway DH, Upton LA. 2016. Predicting the amplitude and hemispheric asymmetry of solar cycle 25 with surface flux transport. J Geophys Res 121(11): 10744-10753. https://doi.org/ 10.1002/2016JA023190.

Howard RF. 1991. Axial tilt angles of sunspot groups. Sol Phys 136(2): 251-262. https://doi.org/10.1007/BF00146534.

Hurwitz L. 1960. Eccentric dipoles and spherical harmonic analysis. J Geophys Res 65: 2555. https://doi.org/10.1029/JZ065i008p02555.

Koskela JS, Virtanen II, Mursula K. 2018. Southward shift of the coronal neutral line and the heliospheric current sheet: Evidence for radial evolution of hemispheric asymmetry. A\&A 618: A105. https://doi.org/10.1051/0004-6361/201832609.

Leighton RB. 1964. Transport of magnetic fields on the Sun. Astrophys J 140: 1547. https://doi.org/10.1086/148058.

Leighton RB. 1969. A magneto-kinematic model of the solar cycle. Astrophys J 156: 1. https://doi.org/10.1086/149943.

Mursula K, Hiltula T. 2003. Bashful ballerina: Southward shifted heliospheric current sheet. Geophys Res Lett 30(22): 2135. https://doi.org/10.1029/2003GL018201.

Mursula K, Hiltula T. 2004. Systematically asymmetric heliospheric magnetic field: Evidence for a quadrupole mode and nonaxisymmetry with polarity flip-flops. Sol Phys 224(1-2): 133 143. https://doi.org/10.1007/s11207-005-4977-7.

Nandy D, Choudhuri AR. 2001. Toward a mean field formulation of the Babcock-Leighton type solar dynamo. I. $\alpha$-coefficient versus Durney's double-ring approach. Astrophys J 551(1): 576-585. https://doi.org/10.1086/320057.

Petrie GJD. 2013. Solar magnetic activity cycles, coronal potential field models and eruption rates. Astrophys $J$ 768(2): 162. https://doi.org/10.1088/0004-637X/768/2/162.

Petrie GJD. 2015. Solar magnetism in the polar regions. Living Rev Sol Phys 12(1): 5. https://doi.org/10.1007//rsp-2015-5.

Pulkkinen PJ, Brooke J, Pelt J, Tuominen I. 1999. Long-term variation of sunspot latitudes. A\&A 341: L43-L46.

Sivaraman KR, Gupta SS, Howard RF. 1999. Measurement of Kodaikanal white-light images - IV. Axial tilt angles of sunspot groups. Sol Phys 189(1): 69-83. https://doi.org/10.1023/A: 1005277515551.

Smith EJ, Balogh A. 2008. Decrease in heliospheric magnetic flux in this solar minimum: Recent Ulysses magnetic field observations. Geophys Res Lett 35(22): L22103. https://doi.org/10.1029/2008GL035345.

Virtanen I, Mursula K. 2016. Photospheric and coronal magnetic fields in six magnetographs. I. Consistent evolution of the bashful ballerina. A\&A 591: A78. https://doi.org/10.1051/0004-6361/ 201628096.

Virtanen II, Mursula K. 2010. Asymmetry of solar polar fields and the southward shift of HCS observed by Ulysses. J Geophys Res 115(A9): A09110. https://doi.org/10.1029/2010JA015275. 
Virtanen II, Mursula K. 2014. North-south asymmetric solar cycle evolution: Signatures in the photosphere and consequences in the corona. Astrophys J 781(2): 99. https://doi.org/10.1088/0004637X/781/2/99.

Wang YM. 1996. Nonradial coronal streamers. Astrophys J Lett 456: L119. https://doi.org/10.1086/309871.

Wang YM, Nash AG, Sheeley NR. 1989. Magnetic flux transport on the Sun. Science 245(4919): 712-718. https://doi.org/ 10.1126/science.245.4919.712.

Wang YM, Sheeley NR. 1991. Magnetic flux transport and the Sun's dipole moment: New twists to the Babcock-Leighton model. Astrophys J 375: 761. https://doi.org/10.1086/170240.

Wang YM, Sheeley NR. 1992. On potential field models of the solar corona. Astrophys J 392: 310. https://doi.org/10.1086/171430.
Winch DE. 1968. Eccentric geomagnetic dipole potential. Planet Space Sci 16(11): 1341-1347. https://doi.org/10.1016/0032-0633 (68)90137-2.

Zhao XP, Hoeksema JT, Scherrer PH. 2005. Prediction and understanding of the north-south displacement of the heliospheric current sheet. J Geophys Res 110(A10): A10101. https://doi.org/ 10.1029/2004JA010723.

Zhou X, Smith EJ. 2009. Solar cycle variations of heliospheric magnetic flux. J Geophys Res 114(A3): A03106. https://doi.org/ 10.1029/2008JA013421.

Zieger B, Virtanen I, Mursula K. 2019. Spatial power spectrum of the photospheric magnetic field during solar minimum. A\&A 623: A51. https://doi.org/10.1051/0004-6361/201834216. 


\section{Appendix}

\section{Potential of a shifted zonal dipole on the solar surface}

For completeness, we derive the magnetic potential of an eccentric dipole on the solar surface. We use a different method of derivation than those given by Hurwitz (1960) or Winch (1968), but we verified that our method led to the same solution.

Let us derive the photospheric potential of a zonal multipole of degree $l$ that is shifted from the center of the Sun by $\boldsymbol{\rho}=\xi \hat{\boldsymbol{x}}+\eta \hat{\boldsymbol{y}}+\zeta \hat{\boldsymbol{z}}$. The potential of a zonal multipole of degree $l$ located at the center of the Sun has the following form in spherical coordinates:

$$
U(\boldsymbol{r})=R_{S}\left(\frac{R_{S}}{r}\right)^{l+1} g_{l}^{0} P_{l}^{0}(\cos \theta)
$$

where $R_{S}$ is the radius of the Sun and $P_{l}^{0}$ is the associated Legendre function. Normalizing the radial distance by $R_{S}$, the potential becomes,

$$
U(\boldsymbol{r})=r^{-(l+1)} g_{l}^{0} P_{l}^{0}(\cos \theta)
$$

First, we evaluate the potential $U$ in Cartesian coordinates on a sphere of unit radius that is shifted by $\boldsymbol{\rho}=\xi \hat{\boldsymbol{x}}+\eta \hat{\boldsymbol{y}}+\zeta \hat{\boldsymbol{z}}$ from the center of the Sun. This can be done by the Taylor series expansion of $U$ :

$$
\begin{aligned}
U(\hat{\boldsymbol{r}}+\boldsymbol{\rho})= & U(\hat{\boldsymbol{r}})+\frac{1}{1 !} \nabla U(\hat{\boldsymbol{r}}) \boldsymbol{\rho}+\frac{1}{2 !} \nabla^{2} U(\hat{\boldsymbol{r}}) \boldsymbol{\rho}^{2}+\frac{1}{3 !} \nabla^{3} U(\hat{\boldsymbol{r}}) \boldsymbol{\rho}^{3}+\ldots \\
= & g_{l}^{0} P_{l}^{0}(\cos \theta)-(l+1) g_{l}^{0} P_{l}^{0}(\cos \theta) \sin \theta \cos \phi \xi-g_{l}^{0} \frac{\partial P_{l}^{0}(\cos \theta)}{\partial \cos \theta} \sin \theta \cos \theta \cos \phi \xi-(l+1) g_{l}^{0} P_{l}^{0}(\cos \theta) \sin \theta \sin \phi \eta \\
& -g_{l}^{0} \frac{\partial P_{l}^{0}(\cos \theta)}{\partial \cos \theta} \sin \theta \cos \theta \sin \phi \eta-(l+1) g_{l}^{0} P_{l}^{0}(\cos \theta) \cos \theta \zeta+g_{l}^{0} \frac{\partial P_{l}^{0}(\cos \theta)}{\partial \cos \theta} \sin ^{2} \theta \zeta+\frac{1}{2 !} \nabla^{2} U(\hat{\boldsymbol{r}}) \boldsymbol{\rho}^{2}+\ldots \\
= & g_{l}^{0} P_{l}^{0}(\cos \theta)-g_{l}^{0} \xi\left[(l+1) \sqrt{1-\cos ^{2} \theta} P_{l}^{0}(\cos \theta)+\cos \theta \sqrt{1-\cos ^{2} \theta} \frac{\partial P_{l}^{0}(\cos \theta)}{\partial \cos \theta}\right] \cos \phi \\
& -g_{l}^{0} \eta\left[(l+1) \sqrt{1-\cos ^{2} \theta} P_{l}^{0}(\cos \theta)+\cos \theta \sqrt{1-\cos ^{2} \theta} \frac{\partial P_{l}^{0}(\cos \theta)}{\partial \cos \theta}\right] \sin \phi \\
& -g_{l}^{0} \zeta\left[(l+1) \cos \theta P_{l}^{0}(\cos \theta)+\left(\cos ^{2} \theta-1\right) \frac{\partial P_{l}^{0}(\cos \theta)}{\partial \cos \theta}\right]+\frac{1}{2 !} \nabla^{2} U(\hat{\boldsymbol{r}}) \boldsymbol{\rho}^{2}+\ldots
\end{aligned}
$$

Using the following recurrence formulae of the associated Legendre function,

$$
\begin{gathered}
\sqrt{1-x^{2}} P_{l}^{m}(x)=\frac{1}{2 l+1}\left[-P_{l+1}^{m+1}(x)+P_{l-1}^{m+1}(x)\right], \\
\sqrt{1-x^{2}} \frac{\mathrm{d}}{\mathrm{d} x} P_{l}^{m}(x)=\frac{1}{2}\left[(l+m)(l-m+1) P_{l}^{m-1}(x)-P_{l}^{m+1}(x)\right], \\
\left(x^{2}-1\right) \frac{\mathrm{d}}{\mathrm{d} x} P_{l}^{m}(x)=-(l+1) x P_{l}^{m}(x)+(l-m+1) P_{l+1}^{m}(x), \\
(l-m+1) P_{l+1}^{m}(x)=(2 l+1) x P_{l}^{m}(x)-(l+m) P_{l-1}^{m}(x), \\
P_{l}^{-m}=(-1)^{m} \frac{(l-m) !}{(l+m) !} P_{l}^{m},
\end{gathered}
$$


the potential $U$ at $\hat{\boldsymbol{r}}+\boldsymbol{\rho}$ becomes,

$$
\begin{aligned}
& U(\hat{\boldsymbol{r}}+\boldsymbol{\rho})=g_{l}^{0} P_{l}^{0}(\cos \theta)-g_{l}^{0} \xi\left[\frac{l+1}{2 l+1}\left[-P_{l+1}^{1}(\cos \theta)+P_{l-1}^{1}(\cos \theta)\right]+\cos \theta \frac{1}{2}\left[l(l+1) P_{l}^{-1}(\cos \theta)-P_{l}^{1}(\cos \theta)\right]\right] \cos \phi \\
& -g_{l}^{0} \eta\left[\frac{l+1}{2 l+1}\left[-P_{l+1}^{1}(\cos \theta)+P_{l-1}^{1}(\cos \theta)\right]+\cos \theta \frac{1}{2}\left[l(l+1) P_{l}^{-1}(\cos \theta)-P_{l}^{1}(\cos \theta)\right]\right] \sin \phi-g_{l}^{0} \zeta(l+1) P_{l+1}^{0}(\cos \theta) \\
& +\frac{1}{2 !} \nabla^{2} U(\hat{\boldsymbol{r}}) \boldsymbol{\rho}^{2}+\ldots \\
& =g_{l}^{0} P_{l}^{0}(\cos \theta)-\frac{1}{2(2 l+1)} g_{l}^{0} \xi\left[\begin{array}{l}
-2(l+1) P_{l+1}^{1}(\cos \theta)+2(l+1) P_{l-1}^{1}(\cos \theta) \\
+(2 l+1) l(l+1) \cos \theta P_{l}^{-1}(\cos \theta)-(2 l+1) \cos \theta P_{l}^{1}(\cos \theta)
\end{array}\right] \cos \phi \\
& -\frac{1}{2(2 l+1)} g_{l}^{0} \eta\left[\begin{array}{l}
-2(l+1) P_{l+1}^{1}(\cos \theta)+2(l+1) P_{l-1}^{1}(\cos \theta) \\
+(2 l+1) l(l+1) \cos \theta P_{l}^{-1}(\cos \theta)-(2 l+1) \cos \theta P_{l}^{1}(\cos \theta)
\end{array}\right] \sin \phi-g_{l}^{0} \zeta(l+1) P_{l+1}^{0}(\cos \theta) \\
& +\frac{1}{2 !} \nabla^{2} U(\hat{\boldsymbol{r}}) \boldsymbol{\rho}^{2}+\ldots \\
& =g_{l}^{0} P_{l}^{0}(\cos \theta)-\frac{1}{2(2 l+1)} g_{l}^{0} \xi\left[\begin{array}{l}
-2(l+1) P_{l+1}^{1}(\cos \theta)+2(l+1) P_{l-1}^{1}(\cos \theta) \\
-\frac{(2 l+1) l(l+1)(l-1) !}{(l+1) !} \cos \theta P_{l}^{1}(\cos \theta)-(2 l+1) \cos \theta P_{l}^{1}(\cos \theta)
\end{array}\right] \cos \phi \\
& -\frac{1}{2(2 l+1)} g_{l}^{0} \eta\left[\begin{array}{l}
-2(l+1) P_{l+1}^{1}(\cos \theta)+2(l+1) P_{l-1}^{1}(\cos \theta) \\
-\frac{(2 l+1) l(l+1)(l-1) !}{(l+1) !} \cos \theta P_{l}^{1}(\cos \theta)-(2 l+1) \cos \theta P_{l}^{1}(\cos \theta)
\end{array}\right] \sin \phi-g_{l}^{0} \zeta(l+1) P_{l+1}^{0}(\cos \theta) \\
& +\frac{1}{2 !} \nabla^{2} U(\hat{\boldsymbol{r}}) \boldsymbol{\rho}^{2}+\ldots \\
& =g_{l}^{0} P_{l}^{0}(\cos \theta)-\frac{1}{2 l+1} g_{l}^{0} \xi\left[-(l+1) P_{l+1}^{1}(\cos \theta)-l P_{l+1}^{1}(\cos \theta)\right] \cos \phi \\
& -\frac{1}{2 l+1} g_{l}^{0} \eta\left[-(l+1) P_{l+1}^{1}(\cos \theta)-l P_{l+1}^{1}(\cos \theta)\right] \sin \phi-g_{l}^{0} \zeta(l+1) P_{l+1}^{0}(\cos \theta)+\frac{1}{2 !} \nabla^{2} U(\hat{\boldsymbol{r}}) \boldsymbol{\rho}^{2}+\ldots \\
& =g_{l}^{0} P_{l}^{0}(\cos \theta)+g_{l}^{0} \xi P_{l+1}^{1}(\cos \theta) \cos \phi+g_{l}^{0} \eta P_{l+1}^{1}(\cos \theta) \sin \phi-g_{l}^{0} \zeta(l+1) P_{l+1}^{0}(\cos \theta)+\frac{1}{2 !} \nabla^{2} U(\hat{\boldsymbol{r}}) \boldsymbol{\rho}^{2}+\ldots
\end{aligned}
$$

Let us now move the center of our coordinate system to $\rho$. Then the potential on the sphere of unit radius is the potential of a zonal multipole that is shifted by $-\boldsymbol{\rho}$ from the center of the sphere. Thus, the potential of a shifted zonal multipole at the solar surface is,

$$
U(\hat{r})=g_{l}^{0} P_{l}^{0}(\cos \theta)-g_{l}^{0} \xi P_{l+1}^{1}(\cos \theta) \cos \phi-g_{l}^{0} \eta P_{l+1}^{1}(\cos \theta) \sin \phi+g_{l}^{0} \zeta(l+1) P_{l+1}^{0}(\cos \theta)+\frac{1}{2 !} \nabla^{2} U(\hat{\boldsymbol{r}})(-\boldsymbol{\rho})^{2}+\ldots
$$

where $\boldsymbol{\rho}=\xi \hat{\boldsymbol{x}}+\eta \hat{\boldsymbol{y}}+\zeta \hat{\boldsymbol{z}}$ is the displacement of the zonal multipole from the center of the Sun.

Now we introduce the Schmidt semi-normalized associated Legendre functions defined as,

$$
\tilde{P}_{l}^{0}(x)=P_{l}^{0}(x)=P_{l}(x) \quad \text { for } \quad \mathrm{m}=0, \quad \text { and } \quad \tilde{P}_{l}^{m}(x)=(-1)^{m} \sqrt{\frac{2(l-m) !}{(l+m) !}} P_{l}^{m}(x) \quad \text { for } \quad \mathrm{m} \neq 0 .
$$

The potential of a shifted zonal multipole becomes,

$$
\begin{aligned}
U(\hat{\boldsymbol{r}})= & g_{l}^{0} \tilde{P}_{l}^{0}(\cos \theta)-g_{l}^{0} \xi(-1)^{-1} \sqrt{\frac{(l+1+1) !}{2(l+1-1) !}} \tilde{P}_{l+1}^{1}(\cos \theta) \cos \phi-g_{l}^{0} \eta(-1)^{-1} \sqrt{\frac{(l+1+1) !}{2(l+1-1) !}} \tilde{P}_{l+1}^{1}(\cos \theta) \sin \phi+g_{l}^{0} \zeta(l \\
& +1) \tilde{P}_{l+1}^{0}(\cos \theta)+\frac{1}{2 !} \nabla^{2} U(\hat{\boldsymbol{r}})(-\boldsymbol{\rho})^{2}+\ldots \\
= & g_{l}^{0} \tilde{P}_{l}^{0}(\cos \theta)+g_{l}^{0} \xi \sqrt{\frac{(l+1)(l+2)}{2}} \tilde{P}_{l+1}^{1}(\cos \theta) \cos \phi+g_{l}^{0} \eta \sqrt{\frac{(l+1)(l+2)}{2}} \tilde{P}_{l+1}^{1}(\cos \theta) \sin \phi+g_{l}^{0} \zeta(l+1) \\
& \times \tilde{P}_{l+1}^{0}(\cos \theta)+\frac{1}{2 !} \nabla^{2} U(\hat{\boldsymbol{r}})(-\boldsymbol{\rho})^{2}+\ldots
\end{aligned}
$$

From the general solution of equation (A.12), we obtain the potential of a shifted zonal dipole $(l=1)$ in terms of the Schmidt seminormalized associated Legendre functions: 


$$
U(\hat{\boldsymbol{r}})=g_{1}^{0} \tilde{P}_{1}^{0}(\cos \theta)+g_{1}^{0} \xi \sqrt{3} \tilde{P}_{2}^{1}(\cos \theta) \cos \phi+g_{1}^{0} \eta \sqrt{3} \tilde{P}_{2}^{1}(\cos \theta) \sin \phi+g_{1}^{0} \zeta 2 \tilde{P}_{2}^{0}(\cos \theta)+\frac{1}{2 !} \nabla^{2} U(\hat{\boldsymbol{r}})(-\boldsymbol{\rho})^{2}+\ldots
$$

For a zonal dipole that is shifted along the symmetry axis $(\xi=0, \eta=0)$, the potential can be written as,

$$
U_{z}(\hat{\boldsymbol{r}})=g_{1}^{0} \tilde{P}_{1}^{0}(\cos \theta)+2 \zeta g_{1}^{0} \tilde{P}_{2}^{0}(\cos \theta)+\frac{1}{2 !} \frac{\partial^{2}}{\partial z^{2}} U(\hat{\boldsymbol{r}})(-\zeta)^{2}+\frac{1}{3 !} \frac{\partial^{3}}{\partial z^{3}} U(\hat{\boldsymbol{r}})(-\zeta)^{3}+\ldots
$$

We can rewrite equation (A.14) in recursive form where each term is calculated from the preceding term,

$$
\begin{aligned}
U_{z}(\hat{\boldsymbol{r}})= & g_{1}^{0} \tilde{P}_{1}^{0}(\cos \theta)-\frac{1}{1 !} \frac{\partial}{\partial z} U(\hat{\boldsymbol{r}}) \zeta-\frac{1}{2 !} \frac{\partial}{\partial z}\left(\frac{\partial}{\partial z} U(\hat{\boldsymbol{r}})(-\zeta)\right) \zeta-\frac{1}{3 !} \frac{\partial}{\partial z}\left(\frac{\partial^{2}}{\partial z^{2}} U(\hat{\boldsymbol{r}})(-\zeta)^{2}\right) \zeta-\ldots-\frac{1}{(l-1) !} \\
& \times \frac{\partial}{\partial z}\left(\frac{\partial^{l-2}}{\partial z^{l-2}} U(\hat{\boldsymbol{r}})(-\zeta)^{l-2}\right) \zeta-\ldots
\end{aligned}
$$

Now we make use of equations (A.3) and (A.9), where it was shown that,

$$
\left.\frac{\partial}{\partial z}\left(r^{-(l+1)} g_{l}^{0} P_{l}^{0}(\cos \theta)\right)\right|_{\hat{r}}=-g_{l}^{0}(l+1) P_{l+1}^{0}(\cos \theta)
$$

Inserting equation (A.16) into equation (A.15) recursively, the photospheric potential of a zonal dipole shifted in the $\hat{\mathbf{z}}$ direction becomes,

$$
U_{z}(\hat{\boldsymbol{r}})=g_{1}^{0} \tilde{P}_{1}^{0}(\cos \theta)+g_{1}^{0} \sum_{l=2}^{\infty} \frac{l !}{(l-1) !} \zeta^{l-1} \tilde{P}_{l}^{0}(\cos \theta)=g_{1}^{0} \sum_{l=1}^{\infty} l \zeta^{l-1} \tilde{P}_{l}^{0}(\cos \theta)
$$

Thus, the Gauss coefficients of a dipole shifted by $\zeta$ along the symmetry axis are the following:

$$
g_{l}^{0}=g_{1}^{0} l \zeta^{l-1}
$$

which is used in the two-dipole model of equation (1).

Cite this article as: Zieger B \& Mursula K. 2020. Two-dipole model of the asymmetric Sun. J. Space Weather Space Clim. 10, 40. https://doi.org/10.1051/swsc/2020041 